\title{
La fusión de cooperativas
}

\author{
Cristina Cano Ortega ${ }^{1}$
}

Recibido: 3 de octubre de 2017 / Aceptado: 20 de febrero de 2018

Resumen. Las cooperativas, especialmente las del sector agroalimentario, tienen un problema de atomización. Necesitan ganar dimensión para ser más competitivas. Entre las diferentes vías de integración a su alcance se encuentra la fusión. La fusión es el proceso mediante el cual dos o más sociedades que se extinguen, todas o algunas de ellas, se integran en una única sociedad mediante la transmisión en bloque por sucesión universal de sus patrimonios y el paso de los socios a la sociedad resultante de nueva constitución o absorbente.

En primer lugar, el presente trabajo analizará las diversas formas de establecer el régimen jurídico de la fusión de cooperativas que han sido desarrolladas por países de nuestro entorno y también norteamericanos (regulación en las propias leyes cooperativas, remisión al régimen del resto de sociedades mercantiles, ley propia para la fusión de cooperativas, etc.). Posteriormente, se centrará en el estudio del régimen jurídico escogido por el ordenamiento español, viendo cómo ha evolucionado tanto la normativa estatal como autonómica sobre la regulación de esta figura, y cómo nos ha llevado a la situación actual de multiplicidad de leyes cooperativas existentes (una estatal y una por cada Comunidad Autónoma), lo que puede dificultar los procesos de integración en general y el de la fusión en particular. Ante este problema se propondrán diferentes soluciones (aplicación supletoria de la ley estatal, una ley armonizadora, aplicación de la Ley de Modificaciones Estructurales, crear una ley que regule únicamente la fusión supra-autonómica de cooperativas, la coordinación de la legislación existente, etc.). Por último, se hará un pequeño repaso al procedimiento de fusión que, como se comprobará, presenta pocas diferencias con la regulación previstan en la Ley sobre modificaciones estructurales, indicando cuáles son los distintos tipos de fusiones que podemos encontrar y cuáles son las diferentes fases del procedimiento que se deben seguir para que la fusión despliegue todos sus efectos.

Palabras clave: Concentración; Integración; Sociedades; Procedimiento; Economía social. Claves Econlit: P13; Q13; G34; K22.

\section{[en] The merger between cooperatives}

Abstract. Cooperatives, especially in the agri-food sector, have a problem of atomization. They need to gain dimension to be more competitive. Among the different integration ways it is the merger. The merger is the process by which companies that are extinguished, all or some of them, are integrated into a single company by means of the block transfer by universal succession of their patrimonies and the passage of the partners to the society resulting of new constitution or the absorbent.

Firstly, the present paper will analyze the different ways to establish the legal regime of the merger of cooperatives that have been developed by neighboring and also North American countries (regulation in the cooperative laws themselves, referral to the regime of the rest of companies, own law for the

$1 \quad$ Universidad de Almería, España

Dirección de correo electrónico: cristinacano@ual.es 
merger of cooperatives, etc.). Subsequently, it will focus on the study of the legal regime chosen by the Spanish legal system, seeing how both state and autonomous regulations have evolved over the regulation of this figure, and how it has led us to the current situation of multiple existing cooperative laws (one national and one for each Autonomous Community), which can hinder integration processes in general and the fusion in particular. Faced with this problem, different solutions will be proposed (supplementary application of the state law, a harmonizing law, application of the Structural Modifications Act, creating a law that only regulates the supra-autonomous fusion of cooperatives, the coordination of existing legislation, etc.). Finally, a brief review will be made of the merger procedure, which, as will be verified, presents few differences with the regulation foreseen in the Structural Modifications Act, indicating which are the different types of mergers that can be found and which are the different phases of the procedure that must be followed so that the merger displays all its effects.

Keywords: Amalgamation; Integration; Enterprise; Procedure; Social economy.

Sumario. 1. Introducción. 2. Los distintos modelos en Derecho comparado. 3. Evolución de la regulación de la fusión de cooperativas en las leyes españolas. 4. Efectos y naturaleza de la fusión. 5. Modalidades de fusión. 6. El procedimiento de fusión. 7. Conclusiones. 8. Referencias bibliográficas.

Cómo citar: Cano Ortega, C. (2017) La fusión de cooperativas. REVESCO. Revista de Estudios Cooperativos, Monográfico, No 126, pp. 94-117. DOI: 10.5209/REVE.59767.

\section{Introducción}

La fusión puede ser definida como aquel proceso mediante el cual, dos o más sociedades que se extinguen, bien todas ellas o bien sólo algunas de ellas, dependiendo de la modalidad de fusión, se integran en una única sociedad a través de la transmisión en bloque por sucesión universal de sus patrimonios y el paso de los socios a la sociedad resultante, ya sea de nueva creación o una de las propias sociedades que se fusionan. Si bien tradicionalmente no ha sido la vía de integración cooperativa más utilizada, sino que lo eran las cooperativas de segundo grado (puede verse, Alfonso, 2000 y 2001), en los últimos años se ha producido un gran impulso de esta vía debido a la Ley 13/2013, de fomento de la integración de cooperativas y de otras entidades asociativas de carácter agroalimentario y por la influencia del modelo de cooperativas de los países del norte de Europa, en los que existe una mayor predisposición a las fusiones y adquisiciones de empresas frente a otros modelos de crecimiento. El presente artículo analizará diversos modelos regulatorios de la fusión de cooperativas en Derecho comparado; la evolución de la normativa sobre esta materia tanto a nivel estatal como autonómico; los efectos, naturaleza y clases de fusiones; y por último, repasará brevemente el procedimiento de fusión contemplado en nuestra normativa.

\section{Los distintos modelos en Derecho comparado}

La regulación de la fusión de las cooperativas en los distintos países de la Unión Europea se ha visto afectada por efecto de las Directivas comunitarias sobre fusiones de sociedades anónimas, ya sea porque, como ahora veremos, el régimen general de fusiones de las sociedades de capital, que ha sido debidamente armonizado en el ámbito comunitario, le es directamente aplicable o porque en la 
propia normativa de la fusión de las cooperativas se percibe la influencia ejercida por estas Directivas debido a la creciente tendencia hacia el modelo economicista, el cual aproxima cada vez más el régimen de las cooperativas al de las sociedades de capital. Así, por regla general, el régimen jurídico de la fusión en cualquier país, independientemente del tipo social de que se trate, tiene en común que se prevén las dos modalidades básicas de fusión, los efectos básicos que produce, un procedimiento similar, etc. Aparte de todas estas notas comunes, en la regulación de la fusión nos podemos encontrar con distintos modelos en los diferentes países europeos y norteamericanos ${ }^{2}$. Pasamos a continuación a analizar brevemente cada uno de ellos.

\subsection{Regulación en la propia normativa cooperativa}

El primero de ellos sería aquel en el que se prevé el régimen jurídico de la fusión dentro de la propia normativa de las cooperativas, como es el caso español. Dentro de este modelo se encuentran por ejemplo Portugal o Canadá.

En el caso de Portugal, el régimen jurídico de las cooperativas se contempla en el Código Cooperativo, aprobado por la Ley núm. 119/2015, de 7 de septiembre, que prevé un estatuto general regulador de todas las cooperativas con independencia de su grado. No obstante, se aplica preferentemente la regulación particular contenida en otras normas para los diferentes sectores de actividad, por lo que el Código se aplica con carácter supletorio. Aunque el legislador portugués, no incluye claramente a las cooperativas dentro del orden civil o mercantil (Montolío, 2000), remite en el propio Código a la legislación mercantil, y en concreto, a la legislación de sociedades anónimas para integrar las lagunas de la legislación propia de las cooperativas (art. 9). El Código contempla de forma breve el régimen jurídico de la fusión de las cooperativas en el artículo 109. Por su parte, el Código das Sociedades Comerciais de 1986 regula la fusión en los artículos $97^{\circ}$ a $117^{\circ}$.

Por lo que respecta a Canadá, el marco normativo es parecido al español ${ }^{3}$. Nos encontramos con una ley de cooperativas estatal (Canada Cooperatives Act, 1998) - que para tratar de igualar las condiciones de la participación de las cooperativas en el mercado al resto de operadores hace una interpretación relajada de los principios cooperativos (Petrou, 2013) - y además cada provincia y los territorios poseen su propia ley de cooperativas. A esto hay que añadir las leyes sobre cooperativas de crédito de las distintas provincias y territorios e incluso la regulación de una nueva figura en alguna provincia como la New Generation Cooperatives Act de Saskatchewan de 1999. Sin embargo, el criterio de aplicación de las distintas leyes es menos conflictivo que el español, pues tratándose de un criterio territorial, la ley estatal se aplicará en el caso de que la cooperativa lleve a cabo su actividad en dos o más provincias y tenga un lugar fijo de negocios en más

2 Para conocer algún detalle de la regulación de las cooperativas en Latinoamérica y en especial sobre la «Ley Marco para las Cooperativas de América Latina» puede consultarse Cracogna, 2013.

3 Otro caso interesante es el de Estados Unidos en el que no existe una Ley de cooperativas aplicable en todo el país, sino que las cooperativas se constituyen de acuerdo con la legislación de cada Estado. Todos los Estados tienen su propia Ley. Aunque las leyes divergen en cuanto a su contenido, se asientan sobre unos principios comunes (Czachorska-Jones, 2013). 
de una provincia, en cualquier otro caso se aplicará la normativa provincial o territorial correspondiente. Por otra parte, por lo que se refiere al derecho supletorio, al tratarse de un sistema basado en el common law -a excepción de Quebec-, en caso de laguna legal serán aplicables las resoluciones dictadas por los Tribunales. Además, la Canada Cooperatives Act en su artículo 3 (4) dispone que, por ejemplo, las disposiciones de la Canada Business Corporations Act o la Canada Corporations Act no se aplicarán a una cooperativa. Si tomamos como ejemplo dos leyes cooperativas, como la estatal (Canada Cooperativas Act, 1998) y la ley de Saskatchewan (The Co-operatives Act, 1996) por tener especial importancia en esta provincia las cooperativas agrícolas, ambas regulan la fusión en unos pocos artículos (arts. 295 a 300 y 151 a 155 respectivamente) con un contenido similar, aunque con algunas diferencias entre ellas.

\subsection{Remisión a la normativa de otras sociedades}

El segundo modelo es en el que, aun existiendo una regulación específica para las cooperativas, en cuanto a la fusión remiten a las leyes de sociedades de capital o mercantiles en general, aunque puedan preverse determinadas especialidades para las cooperativas en estos casos. Es el caso de Italia o Alemania.

Respecto a Italia, el régimen jurídico de las cooperativas se incluye en el Codice civile, específicamente en el Libro Quinto, Título VI, Capítulo I «Delle società cooperative», a lo que hay que sumar la normativa existente por sectores de actividad que se aplicará con preferencia. No obstante, el Codice civile sólo regula aspectos muy concretos de las cooperativas y, por lo demás, remite a la normativa de otros tipos societarios (art. 2.519). En los artículos 2.511 y siguientes no se contempla la fusión de sociedades cooperativas, por lo que, basándonos en el artículo 2.545-nonies que remite expresamente para la fusión y escisión de la sociedad cooperativa al régimen general de sociedades, serán aplicables los artículos 2.501 y siguientes. El régimen jurídico de la fusión recogido en el Codice civile es similar al español para las sociedades mercantiles debido al efecto armonizador de las Directivas comunitarias sobre fusiones.

En Alemania se ha dotado de una regulación específica a las cooperativas, en concreto, la Ley de Cooperativas alemana de 1 de mayo de 1889 (Genossenschaftsgesetz, en adelante, GenG). A pesar de que no se recoge una declaración expresa sobre el derecho supletorio, debido a que en algunos supuestos concretos se realizan remisiones a la legislación general civil o mercantil, la doctrina ve en ello una predisposición a la aplicación en el caso en que sea necesario de la legislación mercantil (Montolío, 2000). Los artículos que regulaban la fusión de cooperativas en la GenG ( $\$ 93 \mathrm{a}$ - $\S 93 \mathrm{~s})$ fueron derogados, y actualmente se contempla de forma conjunta el régimen jurídico de las diferentes modificaciones estructurales, con independencia del tipo social en la Ley para la transformación (Umwandlungsgesetz, en adelante, UmwG). En concreto, el Segundo Libro ( $\S \S 2-1221$ ) de la $U m w G$ recoge el régimen jurídico de la fusión, dedicando los primeros artículos a las disposiciones generales ( $\S 2-38)$ y otros específicos (§§ 79-98) a regular particularmente el régimen de la fusión con la participación de las cooperativas registradas (con mayor detalle, Münker, 2013). 


\subsection{Aplicación de la regulación del tipo social elegido}

Como otro modelo de regulación, tenemos la posibilidad de que se dé libertad a las cooperativas para adoptar una forma social u otra, lo que conllevará que el régimen jurídico aplicable a la fusión dependa del tipo social elegido. Aquí nos encontramos, con el caso de Francia, Reino Unido o Irlanda.

En Francia el régimen jurídico de las cooperativas está formado por una regulación básica establecida en el Estatuto de la Cooperación aprobado por la Ley 47-1775, de 10 de septiembre de 1947, a la que se añade con preferencia la aplicación de normas específicas de determinados sectores cooperativos (art. 2). Además, en el ordenamiento francés las sociedades cooperativas deben constituirse y estructurarse como cualquier sociedad de capital (Montolío, 2000), por lo que también será aplicable a las cooperativas el Código de Comercio francés. Al no contemplarse previsiones específicas para la fusión de cooperativas en el Estatuto de la Cooperación, deben aplicarse las normas que regulen el tipo societario cuya forma haya adoptado la cooperativa, y si existieran, con carácter preferente, las particularidades previstas en las leyes cooperativas sectoriales ${ }^{4}$. Por su parte, el Code de Commerce regula la fusión en los artículos L236-1 a L236-325.

Por su parte, tanto el Reino Unido como Irlanda se basan en el sistema del common law, por lo que no existe en ellos una verdadera legislación estrictamente cooperativa, sino más bien una pluralidad de normas particulares. No hay una forma jurídica especial obligatoria que deban utilizar las cooperativas para constituirse, sino que éstas pueden adoptar cualquier forma legal, aunque los efectos de su constitución serán diferentes según el tipo social escogido. Sin embargo, en Reino Unido, la Co-operative and Community Benefit Societies Act 2014 contempla una estructura legal específicamente diseñada para cooperativas, por lo que la mayoría de cooperativas se constituyen al amparo de esta Ley como sociedades de responsabilidad limitada, ya que tienen ciertos beneficios fiscales y otros tipos de ventajas [por ejemplo, no necesitan la intervención de los tribunales para fusionarse (Snaith, 2013)], a pesar de que pueden constituirse adoptando otras formas como sociedades de capital (companies) o de personas (partnership). Dicha regulación es flexible, por lo que la naturaleza de la cooperativa dependerá de las disposiciones estatutarias de las que se dote la sociedad. En concreto, las secciones 109 a 111 de la Co-operative and Community Benefit Societies Act 2014 regulan la fusión. La Ley se centra en los aspectos procedimentales para realizar la fusión y poco dice sobre la naturaleza, funcionamiento y consecuencias de tal operación. Lo más relevante parece ser que la operación sea aprobada por las Asambleas Generales por las mayorías requeridas, dando mayor libertad en los demás aspectos, como por ejemplo, la información facilitada a los socios sobre la fusión (Snaith, 2013).

Idéntica situación nos encontramos en Irlanda en la que la regulación sobre cooperativas ha estado tan vinculada a la de Reino Unido, que mantiene la Industrial and Provident Society Act 1893 a 1978 (IPSA, 1893) como ley

4 Por ejemplo, para el caso de las fusiones de cooperativas agrarias sí que existe en el Código Rural y de la Pesca Marítima una regulación sobre la fusión (arts. L526-3 y siguientes).

5 A esto hay que sumarle la regulación reglamentaria de los arts. R236-1 y ss. del Code de Commerce francés. 
fundamental en materia de sociedades cooperativas. Las Secciones 53 a 57 de la IPSA, 1893 regula de forma escueta la fusión de dos o más sociedades registradas.

\subsection{Ley propia de fusión de cooperativas}

Por último, existe la posibilidad de regular en una ley la fusión de cooperativas como sucede en Austria. La Ley de cooperativas de Austria es del año 1873 (Genossenschaftsgesetz, 1873), la cual está influenciada por la Ley alemana de cooperativas y contiene la regulación básica aplicable a cualquier tipo de cooperativas, si bien ciertas clases de cooperativas cuentan con una regulación sectorial (Miribung y Reiner, 2013). Aunque no hay una declaración expresa sobre el derecho supletorio aplicable, en ciertos puntos la Ley realiza una remisión a la legislación mercantil (Montolío, 2000). Además, existe una ley específica sobre la fusión de cooperativas, la Genossenschaftsverschmelzungsgesetz, 1980 (en adelante GenVG) que regula detalladamente el régimen de la fusión por absorción ( $\S 2$ a 12), y, en cambio, sólo dedica un artículo a la fusión por constitución ( $\S$ 13), el cual establece determinadas particularidades para ese tipo de fusión.

\section{Evolución de la regulación de la fusión de cooperativas en las leyes españolas}

El régimen jurídico de la fusión de cooperativas ha cambiado notablemente en unas pocas décadas, tanto desde un punto de vista cuantitativo por el número de preceptos dedicados en las leyes cooperativas a este fenómeno, como por los cambios que se han producido desde un punto de vista substantivo.

\subsection{Etapa preconstitucional}

En una primera etapa, la preconstitucional, las leyes cooperativas ni siquiera contemplaban el régimen de la fusión, como fue el caso de la Ley de cooperativas de 1931. Fue con la Ley de Cooperación de 1942, y su Reglamento de 1943 cuando se dedican las primeras menciones a la fusión en la normativa de las cooperativas, limitándose a reconocer la fusión como competencia de la Asamblea General y la mayoría necesaria para aprobar el acuerdo junto con la aprobación del Ministerio de Trabajo. Mientras tanto se promulgaba la Ley de sociedades anónimas de 1951 que regulaba por vez primera la materia de transformación y fusión de las sociedades anónimas en España.

Volvía a mencionar las mayorías exigidas para aprobar el acuerdo y la necesaria aprobación por parte del Ministerio de Trabajo el Reglamento de Cooperación de 1971, sin ni siquiera referirse a la protección de acreedores y socios disconformes a pesar de que la Ley de Anónimas de 1951 ya lo hacía (Paz Canalejo y Vicent Chuliá, 1994). La Ley General de Cooperativas de 1974 junto con su Reglamento de 1978, estaban claramente influenciados por la normativa de la SA, aunque mantenía una excesiva intervención administrativa y no desarrollaba la perspectiva empresarial de la cooperativa. Esta Ley ya hacía mención al acuerdo de las Asambleas, a los efectos esenciales de la fusión y al destino de los Fondos Sociales 
Obligatorios. El Reglamento de 1978 avanzaba aún más en esta regulación al contemplar la posibilidad del derecho a causar baja del socio disconforme y el derecho de oposición de los acreedores siguiendo el modelo de la LSA de 1951.

\subsection{La primera generación de leyes cooperativas autonómicas tras la Constitución española}

Tras la promulgación de la Constitución española, la poca importancia que se había dado tradicionalmente a las cooperativas, el afán de las Comunidades Autónomas por conseguir competencias y la dejadez del Estado en la defensa de las suyas (Paniagua, 2005 y Gadea et al, 2009), terminan por establecer las bases del complejo marco normativo sobre cooperativas que tenemos hoy en día. Con la nueva organización territorial del Estado creada con la Constitución Española de 1978 que dio lugar al Estado de las Autonomías y al reparto de competencias entre Estado y Comunidades Autónomas, ante el silencio constitucional sobre a quién correspondía la competencia en materia de cooperativas, sin incluirla en las competencias exclusivas del Estado, las Comunidades Autónomas fueran asumiendo paulatinamente las competencias legislativas exclusivas sobre esta materia en sus Estatutos de Autonomía. Comenzó el País Vasco y le siguieron Cataluña, Andalucía, la Comunidad Valenciana y Navarra, que además fueron redactando sus propias leyes cooperativas aunque con un claro posicionamiento ante la extramercantilidad de las cooperativas para no perder la competencia asumida (Gadea, 1999).

La primera fue la Ley 1/1982 de Cooperativas del País Vasco, la cual ya contemplaba algunos aspectos relevantes sobre el procedimiento de fusión, y frente a la cual fue interpuesto un recurso de inconstitucionalidad. La STC 72/1983, de 29 de julio, resolvió tal recurso presentado por el Estado, basándose en los principios de especialidad (singularidad propia de las cooperativas) y de no vaciamiento, admitiendo la competencia de la Comunidad del País Vasco para que la competencia autonómica sobre la materia no quedara vacía de contenido. No llegó a valorar la mercantilidad de las cooperativas y, por tanto, si debía ser asumida como competencia exclusiva del Estado ${ }^{6}$. La Ley de Cooperativas de Cataluña de 1983 y la andaluza de 1985 también contemplaban en parecidos términos a la Ley vasca el régimen jurídico del procedimiento de fusión.

Tras la sentencia del Tribunal Constitucional, se promulgó la Ley de 2 de abril de 1987 General de Cooperativas, la ley más extensa y perfeccionada de las existentes hasta el momento, que reflejaba una tendencia hacia la concepción economicista del cooperativismo. El criterio de aplicación era mucho más amplio que el de la Ley estatal vigente. Se aplicaba de manera supletoria a las leyes autonómicas y era de aplicación directa para las cooperativas con domicilio en el resto de Comunidades sin regulación aún sobre cooperativas e, incluso, era aplicable a las domiciliadas en una Comunidad Autónoma con ley propia que desarrollaran la actividad cooperativizada con sus socios fuera de su Comunidad $\left(\mathrm{DF} 1^{\mathrm{a}}\right)$.

6 Como aportación doctrinal reciente sobre la discutida distribución de competencias entre el Estado y las Comunidades Autónomas en materia de cooperativas puede verse Vargas et al, 2014. 
Esta Ley regulaba con detenimiento la fusión de cooperativas en los artículos 94 a 101, influenciada por la LSA de 1989 debido a que se tramitaron paralelamente. La LGC diferenciaba entre fusión por absorción o fusión con constitución de una nueva sociedad; reconocía la posibilidad de que participara una cooperativa en liquidación; determinaba los efectos esenciales de la fusión; especificaba el contenido mínimo del proyecto de fusión; enumeraba la documentación que debía acompañar a la convocatoria de la Asamblea; exigía ciertos requisitos para el acuerdo de fusión; concretaba los requisitos del balance de fusión y la posibilidad de su impugnación; reconocía el derecho de separación de los socios disconformes y el derecho de oposición a los acreedores; y por último, exigía la elevación de los acuerdos a escritura pública.

A esta Ley estatal de cooperativas, le siguieron una nueva generación de leyes cooperativas autonómicas y las primeras de algunas Comunidades Autónomas que aún no habían ejercido sus competencias sobre esta materia que poco a poco fueron asumiendo. Es indispensable destacar entre ellas la Ley 4/1993, de 24 de junio, de Cooperativas del País Vasco, que fue considerada como la primera ley de cooperativas economicista en España y sirvió como modelo a muchas leyes autonómicas e incluso a la vigente ley estatal. Las leyes cooperativas autonómicas generalmente no habían realizado una regulación demasiado detallada de la fusión ni contemplaban la fusión heterogénea (tampoco lo hacía la Ley General de Cooperativas de 1987). Aun así, por ejemplo, el Texto Refundido de la Ley de Cooperativas de la Comunidad Valenciana de 1998 ha sido considerado como ejemplar por su claridad y brevedad, dedicando un solo artículo a la fusión (Paz Canalejo y Vicent Chuliá, 1994). Por lo que respecta a la Ley de Cooperativas Andaluzas de 1999, desarrollo más el procedimiento de fusión incluyendo las menciones mínimas del proyecto o los requisitos del balance.

\subsection{La ley cooperativa estatal de 1999 y las nuevas leyes autonómicas}

A pesar del creciente número de leyes autonómicas, debía existir a nivel estatal una nueva Ley más moderna que al menos estuviera a la altura de la Ley de cooperativas del País Vasco de 1993 y que adoptara también soluciones previstas en las Directivas comunitarias sobre sociedades de capital. Como consecuencia se aprobó la vigente Ley 27/1999, de 16 de junio, de Cooperativas (en adelante, LCOOP). Es una ley flexible y que, en general, merece una buena consideración. Ha sido utilizada como modelo junto con la ley vasca por el resto de Comunidades Autónomas para reformar o promulgar sus nuevas leyes sobre cooperativas.

Como novedades más importantes destacan la regulación de las fusiones heterogéneas y de la posibilidad de constituir grupos cooperativos. La mayor crítica la merece la redacción dada al artículo que contempla su ámbito de aplicación, pues ha sido más restrictiva que la ley estatal anterior, reservando para la LCOOP actualmente un mero papel residual y supletorio, ya que la Ley se aplicará en Ceuta y Melilla, en las Comunidades que no tenga aún ley propia de cooperativas, y a las cooperativas que desarrollen su actividad cooperativizada en el territorio de varias Comunidades Autónomas, excepto cuando en una de ellas se desarrolle con carácter principal, ocurriendo esto último en la mayoría de los casos. 
Con posterioridad, se han modificado muchas leyes cooperativas autonómicas y se han aprobado algunas nuevas. Podemos destacar la Ley 14/2011, de 23 de diciembre, de Sociedades Cooperativas Andaluzas (en adelante, LSCAND), por ser la más economicistas de todas las promulgadas hasta el momento, con un gran apego a la legislación de sociedades de capital (posibilidad de voto plural, menor obligación de dotación de fondos sociales, otros medios de financiación, etc.), lo cual se ha visto aún más reforzado por su Reglamento de desarrollo (aprobado por el Decreto 123/2014, de 2 de septiembre, en adelante RLSCAND).

Por su parte, la LCOOP dedica un capítulo (Capítulo VII, Título I) a la fusión, escisión y transformación. La fusión se regula en los artículos 63 a 67 de forma muy similar a la prevista en la ley estatal anterior, en cuanto a las modalidades de fusión, contenido del proyecto (aunque exige más menciones), documentación que debe facilitarse con la convocatoria, etc.

Actualmente, nos encontramos con dos tendencias en la regulación de la fusión. La primera de ellas se trata de prever un régimen jurídico detallado de la operación, lo cual sucede en la mayoría de las leyes. La segunda tendencia es la que se da en el resto y consiste en dotar a la fusión de un régimen jurídico muy escueto (por ejemplo, la Ley de Cooperativas de Aragón, la de la Comunidad Valenciana o la Ley Foral de Navarra). Entendemos que el primero de estos dos modelos de regulación, el de la previsión detalla del régimen jurídico, es el más adecuado porque asegura la protección de todos los intereses en juego, si bien sería recomendable introducir la posibilidad de ciertas simplificaciones procedimentales cuando concurran determinados requisitos como ocurre en la Ley 3/2009, de 3 de abril, sobre Modificaciones Estructurales de las Sociedades Mercantiles (en adelante, LME).

Aun así, unas leyes han servido de inspiración a otras, por lo que, aunque podemos encontrar algunas diferencias en algunos aspectos, la mayoría incluso han copiado artículos completos de otras leyes autonómicas o de la estatal. A pesar del esfuerzo de las Comunidades Autónomas por apoyar y promover el cooperativismo, este sinsentido de multiplicidad de leyes cooperativas dificulta innecesariamente los procesos de integración entre cooperativas de distintas Comunidades Autónomas y agravan el problema de atomización presente generalmente en las cooperativas, sobre todo, en algunos sectores como el agroalimentario. Esto termina por conducir en algunos casos hacia la "huida del Derecho cooperativo" (Morillas, 2002), puesto que el complejo marco normativo hace que las cooperativas puedan querer utilizar otras formas de organización empresarial con un régimen jurídico menos problemático.

Un importante referente para las leyes cooperativas más recientes y las venideras, lo constituye la LME, que es aplicable a las fusiones entre sociedades mercantiles inscritas, pero que excluye expresamente su aplicación a las cooperativas que se regirán por su específico régimen legal (art. 2 LME). También debe mencionarse el Anteproyecto de Ley de Código Mercantil que, aunque puede que no se apruebe, tiene un incuestionable valor doctrinal. Sin embargo, las novedades incorporadas respecto a la fusión son escasas debido a lo relativamente reciente que podemos considerar aún la LME. 


\section{Efectos y naturaleza de la fusión}

\subsection{Efectos de la fusión}

Del propio concepto de fusión se deducen cuáles son los efectos esenciales de la operación: a) extinción de las sociedades que participan en la operación, bien todas ellas o las absorbidas, dependiendo de la modalidad utilizada; b) paso de los socios de las sociedades participantes a la resultante; y c) transmisión en bloque por sucesión universal de todo el patrimonio de las sociedades participantes a la sociedad resultante (Sequeira, 1993).

Respecto al primero de los efectos enunciados, no todas las leyes cooperativas usan el término extinción de las sociedades participantes, sino que la mayoría mencionan la disolución sin entrar en liquidación, aunque no sea una expresión correcta. En el caso de fusión por creación de nueva sociedad se extinguen todas las sociedades participantes, mientras que en la fusión por absorción se extinguen la o las sociedades absorbidas. La extinción es un efecto propio de la fusión que no lleva aparejada la liquidación. Este error terminológico puede deberse a que el artículo 45 de la Ley General de Cooperativas de 1974, siguiendo a la LSA de 1951, sí que requería un acuerdo previo de las Asambleas Generales de disolverse (Sequeira, 1993). Pero ese acuerdo de disolución ya no debe adoptarse, por lo que la fusión es un proceso autónomo y la extinción es uno de sus efectos. Sí se refieren a extinción el artículo 70.2 de la Ley de cooperativas de la Comunidad de Madrid o el artículo 107.2 de la del Principado de Asturias. Igualmente, la LME en todo momento hace alusión a sociedades extinguidas (arts. 22 y ss. LME) y la Ley de Sociedades de Capital no menciona la fusión como causa de disolución.

Por lo que se refiere al paso de los socios a la sociedad resultante o transmigración de los socios (Paz Canalejo y Vicent Chuliá, 1994), aunque esté implícito en la regulación de la fusión que hacen la mayoría de las normas de cooperativas, no todas se refieren de forma expresa a este efecto. Así se refieren de forma indirecta al mismo cuando en el contenido del proyecto de fusión deben mencionarse los derechos y obligaciones que se reconocen a los socios de las cooperativas extinguidas en la sociedad nueva o absorbente [por ejemplo, art. 91.2.c) Ley de cooperativas de las Islas Baleares]. Como consecuencia de este efecto, todos los socios pasarán a agruparse en una sociedad única resultante de la fusión, salvo aquéllos que hayan ejercitado su derecho de separación (Gadea et al, 2009).

En el caso de las sociedades de capital, los socios de las entidades que transmiten su patrimonio deben recibir la contraprestación consistente en acciones o participaciones sociales, convirtiéndose en socios de la sociedad resultante. Esta participación en la sociedad resultante debe ser proporcional a su participación en su sociedad de origen, siendo generalmente necesario para ello realizar un aumento del capital social en la sociedad absorbente. Esto se consigue con el tipo de canje. Pero en el caso de las cooperativas, la transmigración de los socios no se lleva a cabo a través de la relación de canje fijada en el proyecto de fusión, sino a través del reconocimiento a cada socio del resultado del valor contable de sus aportaciones de capital en la cooperativa extinguida (Morillas y Feliú, 2002). Esto se debe a que en las cooperativas la posición del socio no está vinculada con la 
aportación patrimonial realizada a la sociedad resultante por parte de las cooperativas que se extinguen, sino que los socios pasan a la sociedad resultante por las aportaciones que realizan al capital de la cooperativa que se extingue, incrementado en su caso por el valor de las reservas repartibles (Gadea et. al, 2009). Además, el aumento del capital social en la sociedad absorbente no es necesario, sino que tiene carácter potestativo si se quiere adecuar más a la nueva realidad patrimonial de la sociedad resultante.

Por último, nos encontramos con el efecto de la transmisión en bloque del patrimonio de las sociedades que se extinguen a la sociedad resultante. La transmisión global es absoluta, afectando tanto a los socios como a los terceros que hubieran contratado con las sociedades que se extinguen en la operación (González-Meneses y Álvarez, 2013). La sociedad absorbente o de nueva constitución asumirá todos los derechos y obligaciones de las sociedades extinguidas y responderá ilimitadamente de todas ellas. Aunque no todas las leyes se refieran a que la transmisión se produce por sucesión universal, así debe entenderse, siendo un efecto esencial de la fusión (Garrido de Palma, 2012 y García, 2013). El procedimiento de fusión es tan riguroso con la protección de los socios y acreedores, por la relevancia de los efectos que produce, como es esta sucesión universal que hace innecesaria la transmisión uno a uno de forma individual de los diferentes elementos que constituyen el patrimonio de las sociedades que se extinguen (Garrigues, 1976). Especial mención debe realizarse a los fondos sociales obligatorios o voluntarios de las cooperativas extinguidas. Los fondos, ya sean repartibles o irrepartibles, se integrarán en los de igual clase (art. 63.3 LCOOP). Por tanto, no se producirá el destino de los fondos previstos para el caso de liquidación y no irán a parar al organismo autonómico correspondiente ni se repartirán entre los socios. Distinto sería el caso en el que la sociedad resultante no fuera una cooperativa, pues se debería aplicar el destino de los fondos sociales previsto en sede de fusiones heterogéneas o si es una fusión entre dos cooperativas pero la sociedad resultante es de otro tipo social, el previsto para transformación.

\subsection{Naturaleza jurídica de la fusión}

Por lo que respecta a cuál es la naturaleza jurídica de la fusión, no existe una postura unánime de la doctrina, pero actualmente parece mayoritario el entendimiento de que se trata de una modificación estructural, y siguiendo la concepción procedimental (González-Meneses y Álvarez, 2013), además se entiende como un proceso estructurado en diferentes fases conectadas y correlativas entre sí que al terminar produce una serie de efectos relevantes que ya hemos estudiado anteriormente (Girón Tena, 1952). La fusión, aunque puede adoptar diversas modalidades, sigue un procedimiento único, que en ocasiones para ciertos tipos societarios ante determinadas situaciones puede verse simplificado.

En el procedimiento de fusión existe un convenio entre las sociedades participantes que justifica la producción de los efectos mencionados. El conflicto doctrinal ha sido en torno a cuál es el momento en el que debe situarse tal convenio, si se halla en el proyecto o en el acuerdo de fusión y, por tanto, desde qué momento las sociedades se encuentran ligadas entre sí alrededor de un contenido negocial inmodificable y del que no pueden separarse sin incurrir en 
incumplimiento. Consideramos que es más adecuado entender el proyecto de fusión como el negocio esencial de la operación. Los administradores actúan fuera de sus competencias de representación orgánica durante la realización del proyecto de fusión, por lo que es necesario el acuerdo posterior de la Asamblea para ratificar el negocio, momento en el que las sociedades quedarán vinculadas (Sequeira, 2009).

La relevancia del proyecto se muestra, por ejemplo, en la exigencia de que no sea modificado por las Asambleas para su aprobación, y en el deber de abstención que recae sobre el Consejo Rector una vez que haya sido aprobado el proyecto por los administradores que deben abstenerse de realizar cualquier acto o celebrar cualquier contrato que pueda obstaculizar la aprobación del proyecto o modificar sustancialmente la proporción de la participación de los socios de las cooperativas extinguidas en la resultante. La producción de los efectos de la fusión queda condicionada a que el proyecto sea aprobado por todas las sociedades dentro del plazo de seis meses desde la suscripción del mismo.

\section{Modalidades de fusión}

A continuación vamos a analizar brevemente los distintos criterios a los que suele atender la doctrina de forma más frecuente para establecer las diversas modalidades de fusión.

\subsection{Fusión propia y fusión por absorción}

Una primera distinción se puede realizar entre fusión propia o fusión por absorción. Es la clasificación más clásica, y se parte del propio concepto de fusión que distingue según sea fusión por creación de una nueva sociedad resultante (fusión pura o propia) o fusión por absorción de una o más sociedades cooperativas que se extinguen por otra ya existente (fusión por absorción). Esta distinción se realiza en todas las leyes cooperativas y se centra en si como resultado de la operación surge o no una nueva entidad diferente a las participantes en el proceso de fusión. Aunque la fusión propia es considerada como la figura básica, realmente en la práctica la más utilizada es la absorción.

En la fusión propia se extinguirán todas las sociedades participantes integrándose sus patrimonios y sus socios en la sociedad de nueva constitución, mientras que en la fusión por absorción las sociedades absorbidas se extinguirán y traspasarán sus patrimonios y socios a la sociedad absorbente. En cualquier caso, el régimen jurídico del procedimiento de fusión es común para ambas modalidades, aunque presenten ciertas particularidades según sea fusión por absorción o por creación de una nueva sociedad resultante. En concreto, se trata de las menciones del acuerdo de fusión, de la escritura pública o de la información suministrada a los socios cuando se publica la convocatoria de la Asamblea. 


\subsection{Fusión entre cooperativas de la misma o distinta clase}

La segunda clasificación diferencia entre fusión entre cooperativas de la misma o distinta clase. Son pocas las leyes cooperativas que se refieren expresamente a la fusión entre distintas clases de cooperativas, quizás porque asumen tal posibilidad sin que sea necesario hacer una mención expresa, si bien alguna ley sí que precisa que la fusión sólo puede realizarse entre cooperativas si sus objetos sociales no son incompatibles (art. 64.1 Ley cooperativas de Aragón, art. 90.1 Ley cooperativas de las Islas Baleares o art. 83.1 Ley de cooperativas de La Rioja).

Un ejemplo es el supuesto de fusión entre una cooperativa agroalimentaria y una cooperativa de crédito, en el que habrá que aplicar lo dispuesto en la Ley 13/1989, de 26 de mayo, de Cooperativas de Crédito y el Real Decreto 84/1993, de 22 de enero (reglamento que desarrolla dicha ley), solicitar la autorización administrativa previa ante la Dirección General del Tesoro y Política Financiera o el órgano competente de la Comunidad Autónoma y, si la entidad resultante fuera una cooperativa de crédito inscribirse en el Registro correspondiente del Banco de España y en su caso en los Registros de las Comunidades Autónomas.

\subsection{Fusiones homogéneas y fusiones heterogéneas}

En tercer lugar, atendiendo al tipo social de las sociedades intervinientes, podemos diferenciar entre fusiones homogéneas y heterogéneas o especiales ${ }^{7}$. Si todas las sociedades participantes son, en nuestro caso, cooperativas, se trata de una fusión homogénea. Si participan sociedades de distinto tipo social, es decir, una o más cooperativas con sociedades con otra forma social (por ejemplo, una sociedad cooperativa y una sociedad limitada) o la sociedad resultante es de un tipo social distinto al de las participantes, se trataría de una fusión heterogénea. El régimen jurídico general contemplado en las leyes cooperativas se refiere al supuesto de fusión homogénea, ya que para la fusión heterogénea se dedica un artículo separado. Se pueden distinguir tres modelos regulatorios sobre las fusiones heterogéneas.

El primero de ellos es el de la mayoría de las leyes que permiten la fusión de las cooperativas con cualquier otra clase de sociedad, es decir, con cualquier sociedad civil o mercantil, siempre que no exista una norma legal que lo prohíba [así, por ejemplo, la ley estatal (art. 67), la andaluza (art. 75.6), la valenciana (art. 76) o la catalana (art. 98)]. La sociedad resultante o absorbente puede ser una cooperativa $\mathrm{u}$ otro tipo de sociedad. Estas normas generalmente prevén la aplicación de la normativa de la sociedad absorbente o que se constituya como consecuencia de la fusión, pero con carácter imperativo en cuanto a la adopción del acuerdo y las garantías de los derechos de los socios y acreedores de las cooperativas participantes, se estará a lo dispuesto para la fusión en la ley de cooperativas aplicable. Además, habrá de tenerse en cuenta, en su caso, la legislación sectorial específica aplicable.

El término fusiones “especiales" utilizado por algunas leyes (art. 67 LCOOP) ha sido criticado por la doctrina puesto que no se prevén realmente ninguna peculiaridad procedimental respecto al régimen general de fusiones (Morillas y Feliú, 2002). 
Si la entidad resultante de la fusión no es una sociedad cooperativa, la liquidación de las aportaciones del socio que ejercite el derecho de separación se tendrá que realizar dentro del mes siguiente a la fecha en que se ejercite el mismo por parte de la propia cooperativa de la que se separan. Si la entidad resultante no fuera una cooperativa, el Fondo de Educación y Promoción, el Fondo de Reserva Obligatorio o la parte irrepartible del mismo, así como de cualquier fondo voluntario previsto estatutariamente que tenga el carácter de irrepartible, no pasará a la sociedad resultante, debiendo darse a éstos el destino previsto en caso de liquidación.

El segundo modelo regulatorio sería el de las leyes que restringen la posibilidad de fusión de cooperativas con otras entidades, permitiéndolo solo en el supuesto de que la fusión sea entre cooperativas de trabajo asociado y sociedades laborales, o entre cooperativas agrarias y sociedades agrarias de transformación [Ley del País Vasco (art. 83), la de Castilla y León (art. 85), la de Galicia (art. 78.3) y la de Aragón (art. 64.8)]. Estas normas son doblemente restrictivas, puesto que limitan los sujetos que pueden intervenir y el tipo de sociedad resultante que tiene que ser una sociedad cooperativa tanto en el caso de fusión por absorción como de creación de nueva sociedad. Estas normas prevén que será aplicable el régimen jurídico de la fusión contemplado en las respectivas normas reguladoras de las sociedades que se fusionan, es decir, deberán conciliarse la aplicación de la normativa de las cooperativas participantes y la de las sociedades laborales (Ley 44/2015, de 14 de octubre) o SAT (Real Decreto 1776/1981, de 3 de agosto). Ninguna de estas dos últimas normas prevé el régimen jurídico de la fusión, sí en cambio el Decreto 199/2013, de 23 de julio, sobre las Sociedades Agrarias de Transformación de Cataluña.

El último de ellos es el de las restantes leyes que guardan silencio sobre la posibilidad de que una cooperativa pueda fusionarse con otras entidades que no sean cooperativas (Ley de la Comunidad de Madrid, de las Islas Baleares y de Navarra). En este caso, podría entenderse que no se permiten las fusiones heterogéneas; o que sí es posible pero no está regulada en la legislación autonómica y puede aplicarse supletoriamente la estatal. Es más adecuada esta segunda interpretación por dar un trato más igualitario a las cooperativas de las diferentes Comunidades Autónomas y frente a los demás tipos societarios.

\subsection{Fusiones de ámbito autonómico o supra-autonómico}

Continuando con las siguientes modalidades de fusión, si atendemos al ámbito territorial de las sociedades cooperativas participantes, podemos diferenciar entre fusión de ámbito autonómico o supra-autonómico. La fusión de ámbito autonómico es la regulada en las leyes cooperativas, por lo que no es problemática. Sí lo son, en cambio, las fusiones supra-autonómicas, ya que el legislador -salvo el catalánguarda silencio de forma generalizada sobre qué normas son aplicables en estos casos. Este silencio no debe entenderse como prohibición de este tipo de fusiones, sino simplemente como ausencia de regulación específica. Sería conveniente dotar a estas operaciones de un régimen jurídico adecuado para facilitar que estas fusiones pudieran llevarse a cabo, para que las cooperativas superaran poco a poco su problema de atomización y ganaran dimensión, sin estar condenadas a sólo 
poder crecer dentro de su Comunidad Autónoma o tener que huir hacia otros tipos societarios.

Las propias Comunidades Autónomas no pueden establecer cómo realizar una fusión supra-autonómica, ya que estarían invadiendo competencias de otras Comunidades Autónomas y del Estado (Morillas, 2002). Si el Estado regulara este tipo de fusiones podría producirse igualmente una invasión de las competencias autonómicas pues la operación afecta a la posición de socios o de terceros y es competencia de las normas autonómicas. No obstante, más que de un conflicto de competencias, parece que estamos ante un vacío de competencias.

Se pueden barajar diferentes soluciones ante este dilema jurídico, algunas más deseables que otras, y también unas más factibles que las demás. Si bien lo ideal sería que el Estado recuperara la competencia exclusiva sobre cooperativas, como es algo que parece impensable a estas alturas, vamos a enumerar simplemente algunas de esas opciones posibles (Cano, 2015):

a) aplicación supletoria de la LCOOP a las fusiones supra-autonómicas en virtud del artículo 149.3 CE, aunque en este caso, más que una norma supletoria, debería contemplarse un régimen sustantivo propio que se aplicara a todas las fusiones supra-autonómicas, sin que las leyes autonómicas pudieran regular en contra de lo dispuesto al respecto en la LCOOP;

b) ley armonizadora de las distintas leyes cooperativas autonómicas en virtud del artículo 150.3 CE, para consensuar un mínimo común en el régimen sustantivo privado de la cooperativas (constitución, organización, funcionamiento, relaciones externas, modificaciones estructurales o extinción), dejando margen a las Comunidades Autónomas que, respetando ese mínimo común, puedan introducir innovaciones relacionadas con aspectos procedimentales, de control administrativo y en el régimen de fomento (Paniagua, 2005);

c) aplicación de la Ley de Modificaciones Estructurales a la fusión de cooperativas -al menos supra-autonómicas- por analogía a otros tipos sociales, como sucede en Alemania con la $\operatorname{Umw} G$, aplicando el régimen general de las modificaciones estructurales a las cooperativas, previendo en su caso, ciertas especialidades en la fusión de éstas;

d) coordinación de la normativa existente de acuerdo con el artículo $149.1 .8^{\mathrm{a}} \mathrm{CE}$ y el artículo 9.11 CC sobre el conflicto de leyes. Sólo el artículo 90.3 de la Ley de cooperativas de Cataluña prevé que ante estas fusiones entre cooperativas inscritas en registros de cooperativas de distintas Comunidades Autónomas, será de aplicación a cada cooperativa el procedimiento de fusión establecido por la normativa de cooperativas por la que se rija. Atendiendo a esta solución, los acuerdos sociales se regirán por la LCOOP o ley autonómica que le sea aplicable, coordinándose aquellos actos unitarios como escritura pública e inscripción en el correspondiente Registro (Paz Canalejo y Vicent Chuliá, 1994);

e) Ley propia sobre fusión de cooperativas, como sucede en Austria con la GenVG, aunque no sea la mejor opción por contar ya con demasiadas leyes cooperativas en nuestro ordenamiento. 


\subsection{Fusión nacional o fusión transfronteriza}

El siguiente criterio a tener en cuenta es de nuevo de ámbito territorial y nos permite diferenciar entre fusión nacional o fusión transfronteriza. En las fusiones transfronterizas se aplicará a cada sociedad la normativa de su Ley personal que viene determinada en el caso de las personas jurídicas por su nacionalidad (art. 9.11 CC en relación con el art. 27.2 LME). La fusión transfronteriza engloba dos subtipos, las fusiones intracomunitarias y las extracomunitarias. Para las primeras la LME sí prevé el régimen jurídico de la fusión transfronteriza de este tipo para cuando las sociedades participantes sean sociedades anónimas, limitadas o comanditarias por acciones, excluyendo expresamente en su artículo 56.1 su aplicación a las fusiones transfronterizas en las que participe una sociedad cooperativa. Para el caso de fusiones intracomunitarias en las que se crea una sociedad supranacional se aplicará en su caso el régimen de la Sociedad Anónima Europea (SE) o de la Sociedad Cooperativa Europea (SCE) (sobre esta última puede verse Alfonso, 2006).

\subsection{Fusión ordinaria 0 abreviada}

Otra clasificación nos lleva a diferenciar entre fusión ordinaria o fusión abreviada. Las fusiones ordinarias serían aquellas que se ajustan estrictamente al procedimiento de fusión. Las fusiones abreviadas son aquéllas que permiten prescindir de alguno de los elementos del procedimiento de fusión, es decir, que flexibilizan el régimen de la fusión, posibilitando su simplificación. Frente a la LME que sí lo permite para el caso de las absorciones de sociedad íntegramente participada o participada al noventa por ciento (arts. 49 a 52) o para los casos de acuerdo unánime de fusión adoptado en junta universal en todas las sociedades (art. 42), las leyes cooperativas no contemplan la posibilidad de simplificación procedimental alguna.

\subsection{Fusión propia o fusión impropia}

Por último, podemos distinguir entre fusión propia o fusión impropia, siendo ésta aquel procedimiento de concentración de empresas que se realiza eludiendo el procedimiento legal contemplado para la fusión (Morillas y Feliú, 2002), como podrían ser el canje de valores, la aportación de rama de actividad como contravalor de un aumento de capital, etc. (González-Meneses y Álvarez, 2013). También se habían considerado como fusiones impropias la cesión global del activo y del pasivo e incluso la absorción de una sociedad íntegramente participada.

\section{El procedimiento de fusión}

Como hemos mencionado, la fusión es un procedimiento de carácter imperativo en el que se suceden una serie ordenada de fases que permiten obtener los efectos de la operación. Tradicionalmente se han distinguido cuatro fases: a) la fase 
preliminar, previa o preparatoria; b) la fase decisoria; c) la fase de pendencia y protección de socios disconformes y acreedores; y d) la fase ejecutoria de la fusión. Asimismo, podría producirse una eventual impugnación de la fusión.

El procedimiento de fusión previsto en las normas cooperativas es muy similar al contemplado en la LME, debido a la gran influencia que el Derecho de sociedades y las distintas Directivas comunitarias han tenido sobre la redacción de las leyes cooperativas. Para realizar un breve estudio del procedimiento de fusión, vamos a comparar el régimen previsto en la LCOOP y en la Ley de cooperativas andaluzas. Como se verá, el gran impacto que ha tenido el modelo economicista sobre la ley andaluza ha hecho que su regulación de la fusión sea un híbrido entre la ley estatal de cooperativas y la LME. Antes de nada, debemos advertir, que podrán participar en una fusión tanto una sociedad en liquidación antes de que haya comenzado el reembolso de las aportaciones del capital social (art. 63.2 LCOOP y art. 75.1 LSCAND) como una sociedad en concurso de acreedores, tanto como solución prevista en convenio como en la propia fase de liquidación.

\subsection{Fase previa}

Por lo que respecta a la fase previa de la fusión, los Consejos Rectores soportan todo el peso de las negociaciones entre las cooperativas participantes, la elaboración del correspondiente proyecto común de fusión, que deberá recoger todas las condiciones de la operación y, así como el informe de los administradores y la consecución, en su caso, de un informe de expertos independientes. Las negociaciones previas carecen de una regulación legal, por lo que caen dentro del ámbito de la autonomía negocial (Largo, 1992). Es indudable la importancia de estas negociaciones que generalmente van a realizar los propios administradores, pues del éxito de éstas y de que se hayan llevado a cabo de una forma cautelosa va a depender en gran medida que la operación se efectúe o no y que produzca los efectos queridos. Poner en marcha una fusión supone una serie de costes económicos y sociales (reputación, información sensible, etc.) ante los que los Consejos Rectores deben responder ante un eventual perjuicio a su cooperativa y socios. Estos acuerdos previos no requieren de formalización, aunque en algunos casos sí se deja constancia escrita de los mismos a través de los "protocolos de fusión" o documentos similares.

Una vez finalizadas estas negociaciones previas, se elabora un proyecto común de fusión, que recoge la información esencial de la operación y que, como advertimos con anterioridad, consideramos el negocio jurídico esencial de la misma. Es calificado como "un documento indispensable para el éxito de la operación" (León y Rodríguez, 2017: 469). El contenido del proyecto de fusión debe respetar las menciones mínimas legales, pero suele ser menos extenso que los posibles protocolos de fusión elaborados anteriormente, al tratarse de un documento más formal y técnico.

El proyecto de fusión debe ser redactado por el órgano de administración de las cooperativas participantes en la operación. El proyecto debe ser común a todas éstas cooperativas, a pesar de que no todas las leyes cooperativas lo especifiquen (sí lo hace por ejemplo, el artículo 60.1 RLSCAND), esto es así, porque las Asambleas Generales de las distintas cooperativas que participen en la fusión debe 
aprobar un mismo contenido como acuerdo de fusión. Los miembros del Consejo Rector no sólo tienen la obligación de redactar el proyecto, sino también de suscribirlo como convenio previo. El artículo 30.1 LME precisa al respecto que si falta la firma de alguno de ellos, se señalará al final del proyecto, con indicación de la causa. Este requisito, similar a lo que sucede en la elaboración de las cuentas anuales (art. 37.2 CCom.), lo entendemos extensible al caso de las cooperativas.

El contenido del proyecto de fusión viene establecido por la legislación cooperativa (art. 63.4 LCOOP y art. 60.1 RLSCAND). Es un contenido mínimo que debe respetarse y que es el siguiente:

a) Identificación de las cooperativas participantes y de la resultante (denominación, clase, ámbito, domicilio y datos identificadores de inscripción en los Registros correspondientes);

b) El sistema para fijar la cuantía que se reconoce a cada socio de las cooperativas que se extingan como aportación al capital de la cooperativa nueva o absorbente computando, en su caso, las reservas voluntarias de carácter repartible;

c) Los derechos y obligaciones que se reconozcan a los socios de la cooperativa extinguida en la cooperativa nueva o absorbente;

d) La fecha a partir de la cual las operaciones de las cooperativas que se extingan habrán de considerarse realizadas, a efectos contables, por cuenta de la cooperativa nueva o absorbente;

e) Los derechos que correspondan a los titulares de participaciones especiales, títulos participativos u otros títulos asimilables de las cooperativas que se extingan en la cooperativa nueva o absorbente;

f) El proyecto de Estatutos de la nueva entidad o de las modificaciones de los de la entidad absorbente, en su caso;

g) La información sobre valoración del activo y pasivo del patrimonio de cada sociedad cooperativa que se transmita a la sociedad resultante;

h) Las posibles consecuencias de la fusión sobre el empleo, así como su eventual impacto de género en los órganos de administración y la incidencia, en su caso, en la responsabilidad social empresarial.

Estas tres últimas menciones mínimas contempladas en la regulación andaluza y no en la estatal han sido copiadas de la LME (art. 31). Por último, los artículos 75.3.II LSCAND y 60.2 RLSCAND hacen una última referencia a la posibilidad de incluir en el proyecto con carácter potestativo la previsión de un periodo de carencia, durante el cual a algunas de las sociedades participantes se les podrá privar del disfrute de ciertos servicios o relevar del cumplimiento de determinadas obligaciones de carácter económico, cuando la situación económica o financiera de algunas de las sociedades que se fusionan sea netamente desigual y las circunstancias de la fusión así lo aconsejen.

Las consecuencias básicas del proyecto de fusión aprobado por los Consejo Rectores son esencialmente cuatro. En primer lugar, el proyecto de fusión deberá ser aprobado en el plazo de seis meses (a un año lo eleva la ley andaluza) desde la fecha del proyecto por las Asambleas generales de todas las cooperativas participantes o este documento quedará sin efecto (art. 63.6 LCOOP y art. 60.4 RLSCAND). En segundo lugar, los administradores de las cooperativas que se fusionan tienen la obligación de abstenerse de realizar cualquier acto o celebrar cualquier contrato que pueda obstaculizar la aprobación del proyecto por parte de 
las Asambleas o modificar sustancialmente la proporción de la participación de los socios de las cooperativas extinguidas en la nueva o absorbente (art. 63.5 LCOOP y art. 60.3 RLSCAND). En tercer lugar, deben convocarse las Asambleas Generales de las respectivas cooperativas para aprobar los acuerdos de fusión; y, en último lugar, debe facilitarse en el momento de la convocatoria ciertos documentos con los que se pretende dar efectividad al derecho de información de los socios cooperativistas. En el caso de las cooperativas, no se exige el previo depósito del proyecto de fusión como sí sucede en la LME (si bien éste ha sido sustituido por la publicación en la página web de las distintas sociedades si las tuvieran), aunque algunos reglamentos autonómicos, como el vasco (art. 61), prevén tal posibilidad.

La convocatoria de las Asambleas Generales se hará siguiendo las normas generales de convocatoria respecto a forma y plazos que se deben respetar. Es esencial que la convocatoria sirva para poner a disposición del socio en el domicilio social la información necesaria sobre la fusión e incluso pedir su envío o entrega gratuitos. Las leyes cooperativas (art. 63.7 LCOOP y art. 62.1 RLSCAND) precisan cuáles son los documentos que se han de facilitar a los socios que, en concreto, son:

a) El proyecto de fusión;

b) Los informes redactados por los Consejos Rectores de cada una de las cooperativas sobre la conveniencia y efectos de la fusión proyectada;

c) El balance, la cuenta de pérdidas y ganancias y la memoria explicativa de los tres últimos ejercicios de las cooperativas que participen en la fusión y, en su caso, los informes de gestión y de los auditores de cuentas;

d) El balance de fusión de cada una de las cooperativas cuando sea distinto del último anual aprobado. Podrá tener la consideración de balance de fusión el último balance anual aprobado, siempre que hubiera sido cerrado dentro de los seis meses anteriores a la fecha de celebración de la Asamblea que ha de resolver sobre la fusión (a ocho meses se refiere el art. 61.1 RLSCAND). El artículo 61.3 RLSCAND precisa que la impugnación del balance de fusión se somete al régimen general de impugnación de los acuerdos sociales, si bien no puede suspender por sí sola la ejecución de la fusión;

e) El proyecto de Estatutos de la nueva cooperativa o el texto íntegro de las modificaciones que hayan de introducirse en los Estatutos de la cooperativa absorbente;

f) Los Estatutos vigentes de todas las cooperativas que participen en la fusión;

g) La identificación de las personas físicas o jurídicas de los consejeros de las sociedades que participan en la fusión y la fecha desde la que desempeñan sus cargos, y en su caso, los mismos datos de quienes vayan a ser propuestos como consejeros como consecuencia de la fusión.

Por su parte, exige el artículo 62.2 RLSCAND que las modificaciones sustanciales del activo o del pasivo producidas en cualquiera de las cooperativas que se fusionan, entre la fecha de redacción del proyecto de fusión y de la celebración de la Asamblea que haya de aprobarla, habrán de comunicarse a la Asamblea de todas las sociedades cooperativas que se fusionan. Será el órgano de administración de la sociedad en la que se haya producido el que deba comunicárselas a los órganos de administración de las demás sociedades para que 
informen a sus respectivas Asambleas. Esta previsión se basa en la contemplada en el artículo 39.3 LME.

\subsection{Fase decisoria}

En la fase decisoria, el acuerdo habrá de ser adoptado por las Asambleas Generales de cada una de las Cooperativas que participen en la operación sin realizar modificaciones en el proyecto de fusión (art. 62.1 RLSCAND, la LCOOP no lo prevé expresamente pero así debe entenderse). Esto se debe a que todas las sociedades participantes deben aprobar las mismas condiciones de la operación fijadas en el proyecto de fusión, por lo que las Asambleas o ratifican el proyecto o no lo aprueban, pero no pueden modificarlo porque si no sería necesario iniciar de nuevo todo el procedimiento.

La generalidad de las leyes cooperativas fijan la mayoría necesaria para aprobar el acuerdo de fusión en dos tercios de los votos presentes y representados (art. 64.1 LCOOP). En cambio, el artículo 33.2 LSCAND exige la mayoría reforzada de tres quintos de las personas asistentes, presentes $\mathrm{o}$ representadas en primera convocatoria, debiendo ser de al menos dos tercios en segunda convocatoria. Los socios que estén disconformes con la fusión deberán votar en contra de la misma (o no votar a favor), siendo posible, dejando constancia de su oposición para facilitar la impugnación del acuerdo o ejercer el derecho de separación. Una vez aprobado el proyecto de fusión por la Asamblea General de cada una de las cooperativas participantes en la fusión, éstas quedan obligadas a continuar el procedimiento de fusión (art. 64.3 LCOOP y art. 62.7 RLSCAND).

La publicidad de los acuerdos de fusión sirve para dar información a socios y terceros de la aprobación de la operación por las Asambleas Generales y para iniciar el cómputo de los plazos para el posible ejercicio de derecho de separación o de oposición. La LCOOP prevé que una vez aprobado el acuerdo de fusión de cada una de las cooperativas debe publicarse en el Boletín Oficial del Estado y en un diario de gran circulación en la provincia del domicilio social (art. 64.2). La mayoría de las leyes cooperativas se pronuncian en parecidos términos, si bien refiriéndose al Boletín Oficial de la Comunidad Autónoma correspondiente. En cambio, algunas leyes ya prevén la posibilidad de una difusión telemática de dichos acuerdos como es el caso de la ley andaluza que permite utilizar como medio de publicidad la sede electrónica de la consejería de la Junta de Andalucía competente en materia de sociedades cooperativas (art. 75.5 LSCAND). Además, el artículo 62.3 RLSCAND posibilita que se sustituya por la notificación individual a los socios y acreedores.

\subsection{Fase de pendencia y protección de socios disconformes y acreedores}

Una vez aprobado el acuerdo de fusión, entramos en la fase tuitiva, en la que deben protegerse los derechos de los socios y terceros que se ven afectados por la operación, en concreto, a través de la posibilidad de ejercer el derecho de separación por parte de los socios o el derecho de oposición por parte de los acreedores. Por lo que se refiere al derecho de separación, se reconoce al socio disconforme con la operación, a diferencia de la LME que no prevé esta posibilidad 
para las sociedades mercantiles. El problema en el caso de las cooperativas es diferenciar entre el derecho de separación y el derecho a causar baja. Parece lógico asimilar ambos conceptos pues parece que el legislador ha utilizado el término de derecho de separación proveniente de otros tipos sociales haciéndolo extensible a las cooperativas cuando se está refiriendo al derecho a causar baja justificada. Pueden ejercer el derecho de separación los socios de cualquiera de las cooperativas participantes en la fusión, sin distinción de si se trata de los de la absorbente o de las cooperativas que se extinguen. Los socios legitimados para poder ejercer el derecho de separación son aquellos que no hayan votado a favor de la fusión (art. 65.1 LCOOP y el art. 62.6 RLSCAND se refiere a socios disconformes). Los socios que así lo deseen ejercerán el derecho de separación mediante escrito dirigido al Presidente del Consejo Rector, en el plazo de cuarenta días desde la publicación del anuncio del acuerdo (art. 65.1 LCOOP). Dicho plazo es más o menos amplio dependiendo de la ley cooperativa aplicable, pero varía entre un plazo de uno o dos meses. La cooperativa resultante será la que asuma la obligación de la liquidación de las aportaciones al socio disconforme en el plazo previsto en la normativa para el caso de baja justificada.

En cuanto al derecho de oposición de los acreedores, dada la transmisión por sucesión universal del patrimonio de las sociedades que se extinguen a la resultante, es una forma de proteger a los acreedores ante la supresión de la necesidad de requerir el consentimiento del acreedor cuando se produce un cambio del deudor de una obligación (art. 1.205 CC). La fusión no puede realizarse si no se aseguran previamente o se satisfacen los derechos de los acreedores que se opongan. El fundamento del derecho de oposición reside en el posible perjuicio que pueda sufrir el acreedor por el cambio de deudor, pero no debe acreditarse que realmente se produce ese empeoramiento de la solvencia del deudor.

Aunque con algunas variaciones según la norma aplicable, generalmente están legitimados para ejercer el derecho de oposición los acreedores de las sociedades participantes en la fusión cuyos créditos hayan nacido antes del último anuncio de fusión (art. 66 LCOOP) o de la publicación del proyecto de fusión (art. 62.5 RLSCAND) y que no se encuentren adecuadamente garantizados. Los destinatarios de la oposición serán las sociedades participantes en la fusión (Escribano, 2009). La fusión no podrá llevarse a cabo antes de que transcurra uno o dos meses (art. 62.5 RLSCAND y art. 66 LCOOP respectivamente) desde la fecha del último anuncio del acuerdo de fusión. Durante ese plazo los acreedores podrán ejercer su derecho de oposición por escrito, dirigido al Presidente del Consejo Rector en línea con lo dicho en el derecho de separación ya que las leyes cooperativas no lo precisan, e identificando su crédito mediante fecha de nacimiento, vencimiento y cuantía (Esteban, 2007). Se trata de un plazo de suspensión durante el cual no podrá efectuarse la fusión hasta que los créditos no sean satisfechos o suficientemente garantizados. La problemática reside en saber qué se entiende por suficientemente garantizados, pareciendo válido el criterio del acreedor para determinar esa suficiencia. Para evitar problemas, será aconsejables una fianza solidaria prestada por una entidad de crédito (art. 62.5 RLSCAND siguiendo el art. 44.3 LME) o incluso el propio pago. 


\subsection{Fase ejecutoria}

Por último, nos encontramos con la fase de ejecución. Una vez que ha transcurrido el plazo necesario para que socios y acreedores hayan podido ejercer sus respectivos derechos, deben ejecutarse los acuerdos. Para ello, éstos deben elevarse a escritura pública "única" -suelen exigir la mayoría de las leyes- e inscribirse en el registro correspondiente, sometiéndose, por tanto, a un control de legalidad. El artículo 62.7 RLSCAND prevé la posibilidad de que la formalización del acuerdo se haga en acta única, si bien en ciertos supuestos tendrá que ser mediante escritura pública (si así lo acuerdan las sociedades cooperativas fusionadas y si se trata de alguno de los supuestos del art. 5.2 RLSCAND).

La escritura pública contendrá de forma íntegra los acuerdos de fusión aprobados por las respectivas Asambleas Generales. Además, si la fusión se realiza mediante la creación de una nueva sociedad, deberán incluirse las menciones exigidas por la ley para la escritura de constitución en cuanto sean aplicables en este caso. En cambio, si la fusión se realiza por absorción la escritura contendrá las modificaciones estatutarias que se hubieran acordado por la cooperativa absorbente. También habrá de contener el balance de fusión de las cooperativas que se extinguen (art. 62.7 RLSCAND y con menos detalle el art. 64.4 LCOOP). Finalmente, deberá manifestarse expresamente que no se ha producido oposición alguna de acreedores o si ésta se ha producido, deberá acreditarse que han sido pagados o garantizados los créditos de los acreedores que se han opuesto. Idénticas declaraciones habrá que realizar sobre si los socios han ejercido su derecho de separación o no.

Por lo que respecta a la inscripción en el registro correspondiente es obligatoria y tiene carácter constitutivo. La inscripción supondrá la cancelación de las sociedades que se extinguen y la modificación de la absorbente o el nacimiento de la nueva cooperativa que se constituya como consecuencia de la operación. En cuanto a la posibilidad de impugnar la fusión, ninguna ley cooperativa prevé un régimen de impugnación específico para la fusión como sí sucede en el artículo 47 LME. No obstante, el artículo 62.9 RLSCAND establece que la impugnación de la fusión se regirá por el régimen general de impugnación de acuerdos sociales. Esta posibilidad debe entenderse extensible al resto de leyes cooperativas, si bien hubiera sido mejor por razones de seguridad jurídica establecer unos motivos tasados y unos plazos más breves para la impugnación siguiendo el ejemplo de la LME.

\section{Conclusiones}

La fusión es una vía de integración adecuada para las cooperativas que quieren ganar dimensión para poder ser más competitivas. Se trata de un procedimiento formal y riguroso que supone unos grandes efectos, ya que conlleva el mayor nivel de concentración posible entre empresas, y cuyo marco jurídico en el caso de las cooperativas se ve complicado por la multiplicidad de leyes cooperativas con las que contamos en nuestro ordenamiento jurídico. Esto dificulta los procesos de fusiones entre cooperativas de diferentes Comunidades Autónomas, si bien, no los 
imposibilita. En el artículo hemos ofrecido diversas alternativas (en algunos casos inspirados por la normativa de otros países) que podría poner en práctica el legislador para solucionar este problema, pero que, actualmente, dado el coste político que supondría la mayoría de ellas dudosamente se llevarán a cabo. Por tanto, la única solución posible a corto plazo pasa por coordinar la aplicación de las diversas leyes cooperativas en los casos de fusiones supra-autonómicas.

Por lo demás, como se ha comprobado, el régimen jurído de la operación es cada vez más similar al régimen previsto antes en la LSA y ahora en la LME, existiendo actualmente pocas diferencias como la no necesidad de una relación de canje o el reconocimiento del derecho de separación del socio disconforme con la operación. Por todo ello, parece que cada vez estaría más justificado (conservando el reconocimiento de esas escasas diferencias señaladas) extender la aplicación del régimen jurídico de la fusión previsto en la LME a las sociedades cooperativas.

\section{Referencias bibliográficas}

Alfonso Sánchez, R. (2006) La Sociedad Cooperativa Europea. Un nuevo tipo social en un escenario complejo. Noticias de la Unión Europea, No 252, pp. 19-34.

Alfonso Sánchez, R. (2001) La integración cooperativa. La cooperativa de segundo grado. En Alonso Espinosa, F.J. (coord.) La sociedad cooperativa en la Ley 27/1999, de 16 de julio, de cooperativas, Granada: Comares, pp. 355-386.

Alfonso Sánchez, R. (2000) La integración cooperativa y sus técnicas de realización: la cooperativa de segundo grado. Valencia: Tirant lo Blanch.

Cano Ortega, C. (2015) La fusión de cooperativas. Madrid: Marcial Pons.

Cracogna, D. (2013) The Framework Law for the Cooperatives in Latin America. En Cracogna, D.; et al. (eds.) International Handbook of Cooperative Law, Berlin: Springer, pp. 165-186.

Czachorska-Jones, B.; Finkelstein, J.G. y Samsami, B. (2013) United States. En Cracogna, D.; et al. (eds.) International Handbook of Cooperative Law, Berlin: Springer, pp. 760 y 763.

Escribano Gámir, R.C. (2009) Derecho de oposición de los acreedores sociales, en Rodríguez Artigas, F. et al. (dir.) Modificaciones Estructurales de las Sociedades Mercantiles. Tomo I, Cizur Menor (Navarra): Aranzadi-Thomson Reuters, pp. 589-644.

Esteban Ramos, L.M. (2007) Los Acreedores Sociales ante los Procesos de Fusión y Escisión de Sociedades Anónimas: Instrumentos de Protección. Cizur Menor (Navarra): Thomson-Aranzadi.

Gadea, E. (1999) Derecho de las cooperativas. Bilbao: Universidad de Deusto.

Gadea, E.; Sacristán, F. y Vargas Vasserot, C. (2009) Régimen Jurídico de la Sociedad Cooperativa del Siglo XXI, Realidad actual y propuestas de reforma. Madrid: Dykinson.

García Sanz, A. (2013) II. Fusión. En Capítulo VIII. Modificaciones Estructurales. En Peinado Gracia, J. I. (dir.) Tratado de Derecho de Cooperativas. Tomo I, Valencia: Tirant lo Blanch, pp. 780-795.

Garrido de Palma, V.M. (2012) La sucesión universal en las modificaciones estructurales. En Garrido de Palma, V.M. (dir.) Modificaciones estructurales y reestructuración empresarial. Valencia: Tirant lo Blanch, pp. 143-156.

Garrigues, J. (1976) Dictámenes de Derecho Mercantil. Tomo III, Madrid. 
Girón Tena, J. (1952) Derecho de Sociedades Anónimas (Según la Ley de 17 de Julio de 1951). Valladolid: publicaciones de los seminarios de la Facultad de Derecho.

González-Meneses, M. y Álvarez, S. (2013) Modificaciones Estructurales de las Sociedades Mercantiles. Madrid: Dykinson.

Largo Gil, R. (1992) La fusión de sociedades mercantiles. Fase preliminar, proyecto de fusión e informes. Madrid: Civitas.

León Sanz, J. y Rodríguez Sánchez, S. (2013) Modificaciones estatutarias y estructurales. En Morillas Jarrillo, M. y Vargas Vasserot, C. Retos y oportunidades de las sociedades cooperativas andaluzas ante su nuevo marco legal. Madrid: Dykinson, pp. 459-502.

Miribung, G. y Reiner, E. (2013) Austria. En Cracogna, D.; et al. (eds.) International Handbook of Cooperative Law, Berlin: Springer, pp. 232-234.

Montolio, J.Mª (2000) Legislación cooperativa en la Unión Europea. Madrid: Ministerio de Trabajo y Asuntos Sociales.

Morillas Jarillo, Mª.J. (2002) El ámbito de aplicación de las leyes de sociedades cooperativas. En Derecho de sociedades: libro homenaje al profesor Fernando Sánchez Calero. Vol. 5. Madrid: McGraw Hill, pp. 4745-4812.

Morillas Jarillo, Ma.J. y Feliú Rey, M.I. (2002) Curso de cooperativas. Segunda edición. Madrid: Tecnos.

Münker, H.-H. (2013) Germany. En Cracogna, D.; et al. (eds.) International Handbook of Cooperative Law, Berlin: Springer, pp. 413-425.

Paniagua Zurera, M. (2005) La sociedad cooperativa. Las sociedades mutuas y las entidades mutuales. Las sociedades laborales. La sociedad de garantía recíproca. Volumen I. En Olivencia, M.; et al. (dir.) Tratado de Derecho Mercantil. Madrid: Marcial Pons.

Paz Canalejo, N. y Vicent Chuliá, F. (1994) Ley General de Cooperativas. En Sánchez Calero y Albaladejo (Dir.) Comentarios al Código de Comercio y legislación mercantil especial. T. XX, vol. 3. Madrid.

Petrou, T. (2013) Canada. En Cracogna, D.; et al. (eds.) International Handbook of Cooperative Law, Berlin: Springer, pp. 289-316.

Sequeira Martín, A. (1993) Transformación, Fusión y Escisión (arts. 223-251). En Sánchez Calero (Dir.) Comentarios a la Ley de Sociedades Anónimas. T. VII. Madrid: EDERSA.

Sequeira Martín, A. (2009) El concepto de fusión y sus elementos componentes. En Rodríguez Artigas, F. et al. (dir.) Modificaciones Estructurales de las Sociedades Mercantiles. Tomo I. Cizur Menor (Navarra): Aranzadi-Thomson Reuters, pp. 375420.

Snaith, I. (2013) United Kingdom. En Cracogna, D.; et al. (eds.) International Handbook of Cooperative Law, Berlin: Springer, pp. 735-758.

Vargas Vasserot, C.; Gadea Soler, E. y Sacristán Bergia, F. (2014) Derecho de las sociedades cooperativas. Introducción, constitución, estatuto del socio y órganos sociales. Madrid: La Ley. 